\title{
Development of Rapid Diagnostic Kit for Identification of Hanwoo (Korean Native Cattle) Brand Meat by Detecting BIO-TAG
}

\author{
Kyung Hoon Baek, Sung Kwon Park¹, Myung Hoon Lee², Sung II Kim, \\ Soo Hyun $\mathrm{Cho}^{1}$, and Chang Bon Choi* \\ School of Biotechnology, Yeungnam University, Gyeongsan 712-749, Korea \\ ${ }^{1}$ National Institute of Animal Resource, RDA, Suwon 441-706, Korea \\ ${ }^{2}$ D\&P Biotech, Daegu 700-422, Korea
}

\begin{abstract}
This study was performed to develop a rapid immuno-assay kit, by using a specific antigen to detect Hanwoo brand meat. We selected a synthetic antigen specific to our target antibody, named BIO-TAG (Tyr-D-Ala-Phe), by utilizing a computerbased analysis and literature review. BIO-TAG tagged with adjuvant was subcutaneously injected in sheep and Hanwoo. The serum and meat juice of the immunized or non-immunized animal were then analyzed, to measure the titer of antibody by ELISA and Western blot. The amount of antibodies against the BIO-TAG increased $(p<0.05)$ in serum by vaccination. Furthermore, meat juice from the immunized Hanwoo showed greater $(p<0.05)$ antibody titer, compared with those from non-immunized groups. To optimze the dilution factor, we performed dot-ELISA, with various combination levels of BIOTAG. Results from dot-ELISA showed that $2 \mathrm{mg} / \mathrm{mL}$ BIO-TAG was sufficient to distinguish the immunized meat from nonimmunized groups. These results support our hypothesis that simple immunization of Hanwoo generates a sufficient amount of antibodies to be detectable in the meat juice by means of the immune-assay. Therefore, specific Hanwoo brand meat can be more precisely identified by our rapid diagnostic kit. This technology can deter possible fraud of counterfeit meat brands in the Korean domestic market with ease and rapidity; and offers a new tool that guarantees consumers high quality Hanwoo brand beef.
\end{abstract}

Keywords: Hanwoo, BIO-TAG, synthetic peptide, rapid diagnosis kit

\section{Introduction}

Meat quality trait and originality are very important factors and this will determine the competitiveness of Hanwoo industry in Korea. In response to this demand, tremendous amount of research has been performed to improve the meat quality of Hanwoo by castration (Kim et al., 1996; Lee et al., 2004; Lee et al., 1997; Sung et al., 1996), modification of feedstuff (Cho and Ahn, 2006; Cho et al., 2000; Song et al., 1998) as well as feed supplements (Paek et al., 2005; Song and Choi, 1994), and determination of slaughter age (Kim et al., 1996; Kim et al., 2005; Lee, 1998). However, Hanwoo industry is still struggling with some issues, especially the untransparent distribution system, despite the continuous efforts of Ko-

*Corresponding author: Chang Bon Choi, School of Biotechnology, Yeungnam University, Gyeongsan 712-749, Korea. Tel: +82-53-810-3023, Fax: +82-53-810-4769, E-mail: cbchoi@yu. ac.kr rean government in monitoring the Hanwoo distribution channels. Origin of meat production is still falsely represented or even omitted; cases reported forging and omitting labels increased from 313 in 2006 to 717 cases in 2010 and 373 cases as of May 2012 (National Agricultural Quality Service, Korea). Therefore, rather than just rely on the monitoring system, it will be plausible to develop the rapid diagnostic kit for everyone can simply use on site to detect the authentic Hanwoo brand meat from counterfeits.

Hanwoo and Holstein can recently be distinguished by DNA analysis (Chung and Chung, 2004). This method, however, demands expensive equipment, a long period of time, and many specialists for its adequate operation, which hampers its employment in the field. Morever, DNA analysis cannot differentiate the specific brand meat among Hanwoo products.

It is necessary to develop a quick and accurate diagnostic technology for identifying the specific brand meat on site for sustainable development of Hanwoo industry in 
Korea. Objective of the current study was to develop the diagnostic kit utilizing the synthetic peptide (BIO-TAG) to discriminate the specific Hanwoo brand meat, therefore protecting Hanwoo industry in Korea. The hypothesis of this study is that immunization of specific brand Hanwoo with antigen may let us discriminate and trace the specific Hanwoo brand meat by detecting the antibody against the immunized antigen after slaughter or even in distributed into the market. Our results show the possibility of detecting the antibodies against specific antigens from immunized animals as well as discriminating the immunized meat from non-immunized meat.

\section{Materials and Methods}

\section{Selection \& synthesis of BIO-TAG (synthetic pep- tide)}

Epitopes in selected peptide sequences showing low genetic correlations with cattle (i.e., crab, cockroach, or frog) were analyzed for their antigenic index, surface probability, hydrophilicity and flexible region based on the amino acid sequence by using the AntheProt program (Release 2.0, C.Geourjon \& G. Deleage, Institute of biology and chemistry of proteins, France) (Benkirane et al., 1995; Briand et al., 1997; Kramer et al., 1998; Lee and Watson, 2002; Mohri et al., 1997; Tooyama et al., 2000). We then performed the MHC binding prediction, processing prediction and immunogenicity prediction analysis and linear epitope prediction and discotope prediction to promote immune reaction of $\mathrm{T}$ cells and $\mathrm{B}$ cells, respectively, by using the web based tools (IEDB and Analysis Resource, 2012). By performing the epitope conservancy, epitope cluster analysis, and homology mapping, we selected the peptide sequence, Tyr-D-Ala-Phe, as an optimal antigen. The Tyr-D-Ala-Phe is a N-terminal sequence commonly found in a peptide family including the dermophin and deltorphin (Tooyama et al., 2000). This peptide sequence was named as a 'BIO-TAG' in this study.

BIO-TAG was synthesized by Peptron Inc. (Korea). Since the purpose of this study is to produce antibodies specific for certain antigen, all peptides were generated in a D-form which T-cell immune response is greater than Lform peptides (Van Regenmortel and Muller, 1998). BIOTAG was attached with carrier protein due to its small size (3 mer). It was attached with KLH (Keyhole Limpet Hemacyanin) for vaccination and with ovalbumin (45 kDa protein) for ELISA, Western blotting, and Dot-ELISA analysis.

\section{Screening analysis for detecting the antibody against the BIO-TAG}

Exraction of antibody specific for BIO-TAG was conducted in sheep, which is another ruminant animal other than Hanwoo following the Baek et al.'s (2007) immunization protocol. BIO-TAG diluted in saline $(100 \mathrm{mg} / \mathrm{mL}$ and $10 \mathrm{mg} / \mathrm{mL}$, for high and low concentration, respectively) was mixed 1:1 with adjuvant (Freund's incomplete, Sigma, USA), and $2 \mathrm{~mL}$ solution was then subcutaneously injected three times at $3 \mathrm{wk}$ intervals into 2 sheep that were bred at the ranch of Yeungnam University. Immunized or non-immunized serum for antibody detection were collected according to Choi et al.'s method (2008). Briefly, blood sample was collected from jugular vein before and $12 \mathrm{~d}$ after injection, incubated for $1 \mathrm{~h}$ at RT, and centrifuged at 2,800 rpm for $30 \mathrm{~min}$ to separate the immunized and non-immunized serum. Muscle samples $(20 \mathrm{~g})$ were biopsied from biceps femoris and snap-frozen. Immunized and non-immunized meat juice was extracted from muscle samples by thawing them at RT and stored at $-20^{\circ} \mathrm{C}$ until analysis.

Concentration and activity of extracted antibodies were tested by ELISA following the procedure of Choi et al. (2008). Primary antibodies including immunized and nonimmunized meat juice were diluted into 1:3,000, 1:9,000, $1: 27,000$ and 1:81,000 and secondary antibody (anti-sheep IgG; Sigma A-5187, USA) were diluted into 1:10,000.

Antibody specificity for BIO-TAG was also analyzed by Western blot analysis following the procedure of Baek et al. (2005). Ten mg of ovalbumin conjugated BIO-TAG was run on 4 polyacrylamide gel (SDS-PAGE) and transferred onto 4 nitrocellulose membranes. Membranes were then incubated with diluted primary antibodies $(1: 10,000)$ followed by secondary antibody (anti-sheep IgG; Sigma A-5187, USA), washed with PBS and developed with alkaline phosphatase substrate. All 4 membranes were developed in the dark room with same amount of exposure time.

\section{Research for screening Hanwoo brand meat}

A total of 3 Hanwoo in the late fattening period (1 mon. pre-slaugher) was subcutaneously injected with $1 \mathrm{~mL}$ antigen containing $1 \mathrm{mg} / \mathrm{mL}$ BIO-TAG. The amount of antigen injected was determined by body weight of cattle. Imject Alum (Pierce 77161, USA) was used as an immunopotentiator instead of Freund's adjuvant due to the issue of adjuvant residues in animals.

Four wk after BIO-TAG injection, 3 Hanwoo was slaughtered. Immunized and non-immunized serum was iso- 
lated and saved for ELISA analysis. For sampling the immunized meat juice, $300 \mathrm{~g}$ of fore shank, hind shank and loin muscle tissue was collected. Commercial meat purchased from butcher shop was used as a non-immunized control. Immunized and non-immunized meat juice of Hanwoo was extracted by the same method used in sheep and analyzed by ELISA for detecting the production of antibody against BIO-TAG. In order to confirm the data from ELISA analysis, immunized meat juice from the loin sample was screened by Dot-ELISA. Immunized serum was used as a positive control and non-immunized juice was as a negative control.

Dot-ELISA was performed following the method of Macedo-Silva et al. (2000) with slight modification. Briefly, different concentration $(2,0.2,0.02 \mathrm{mg} / \mathrm{mL})$ of ovalbumin conjugated BIO-TAG (5 mL each) was dotted onto the nitrocellulose membrane, incubated overnight at $4^{\circ} \mathrm{C}$ and blocked with 5\% BSA/TBS-T. Immunized serum (positive control), non-immunized meat juice (negative control) and meat juice were used as a primary antibody and their dilution factors were all 1:1,000. Immunized meat juice was diluted into 1:2,000 to screen the optimum condition of antigen detection. Anti-bovine IgG/HRP (Sigma A-8917, USA) was used as a secondary antibody. Memrane was washed with TBS-tween and visualized by using the 4-chloro-1-naphthol.

\section{Statistical analysis}

Data were analyzed using SAS software (SAS Institute, USA, 2008), and Duncan's multiple range test was used to compare differences between means. Significance was defined at $p<0.05$.

\section{Result and Discussion}

\section{Detection of antigen against BIO-TAG}

Titers of serum and meat juice from sheep immunized with the BIO-TAG were compared with non-immunized serum by ELISA analysis (Fig. 1 and 2). Antibodies against the BIO-TAG were increased (maximum $80.24 \%$ increase, $p<0.05$ ) in serum by immunization with both levels of BIO-TAG $(100 \mathrm{mg} / \mathrm{mL}$ and $10 \mathrm{mg} / \mathrm{mL})$ compared to those in serum before immunization. This result is concomitant with Choi et al. (2010) data indicating that synthetic peptide (BIO-TAG) can be used as an appropriate antigen which generate enough amount of antibodies against BIO-TAG in our current study.

Although antibody titers in meat juice were lower than serum, meat juice immunized with BIO-TAG (both in 1:

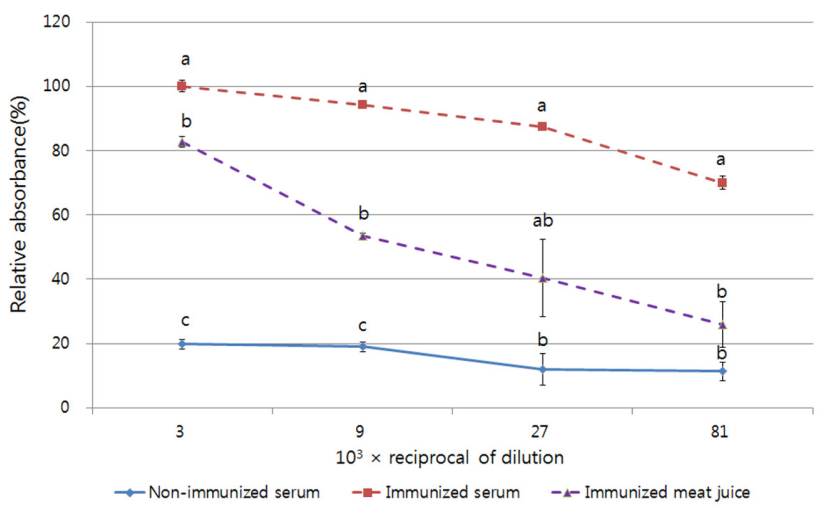

Fig. 1. Reactivity of non-immunized serum, immunized serum and meat-juice in sheep against $100 \mathrm{mg} / \mathrm{mL}$ BIO-TAG (synthetic peptides) as detected by ELISA. The relative reactivity was expressed as percentage of the maximum optical density of sheep immunized serum. Error bars are expressed as means \pm S.E. for 3 time repeats. Values with different letters $\mathrm{a}, \mathrm{b}$ or $\mathrm{c}$ are significantly different among 3 treatments (non-immunized serum, immunized serum and immunized meat juice) by the same dilution rate $(p<0.05)$.

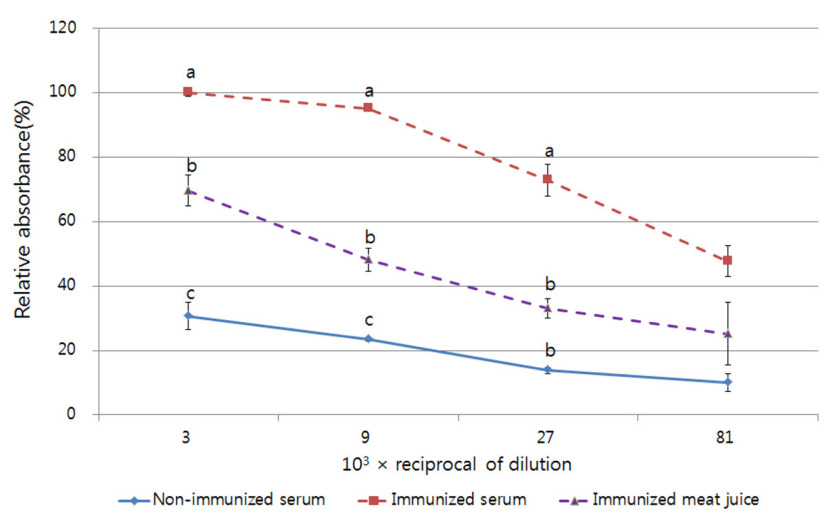

Fig. 2. Reactivity of non-immunized serum, immunized serum and meat-juice in sheep against $10 \mathrm{mg} / \mathrm{mL}$ BIO-TAG (synthetic peptides) as detected by ELISA. The relative reactivity was expressed as percentage of the maximum optical density of sheep immunized serum. Error bars are expressed as means \pm S.E. for 3 time repeats. Values with different letters $\mathrm{a}, \mathrm{b}$ or $\mathrm{c}$ are significantly different among 3 treatments (non-immunized serum, immunized serum and immunized meat juice) by the same dilution rate $(p<0.05)$.

3,000 and 1:9,000 dilution) produced greater $(p<0.05)$ amount of antibodies compared to non-immunized meat juice. This is basic and meaningful result of the current study providing us clear possibilities that antibodies can be detected both in serum and in meat juice by BIO-TAG immunization in Hanwoo.

Furthermore, the level of antibodies produced by immunization of $10 \mathrm{mg} / \mathrm{mL}$ antigen was similar to that produced 
by $100 \mathrm{mg} / \mathrm{mL}$ antigen in sheep. This data indicate that smaller amount of antigen can induce enough immune response and therefore allows us to reduce the production cost of the synthetic peptide (BIO-TAG). Nargi et al. (1999) has also proved this concept showing that small amount of antigen composed of D-form amino acids can produce enough amount of antibodies which can induce immune response. Further in vitro and in vivo research for screening the appropriate amounts of antigens as well as antigenic substances (i.e., Carrier proteins, adjuvant and otherwise) will be necessary to establish the optimal immune condition.

Expression of BIO-TAG was by using Western blot analysis. No band was detected in non-immunized serum (Fig. 3(A)) or meat juice (Fig. 3(B)). Strong immune reaction was detected both in immunized serum and meat juice at $45 \mathrm{kDa}$. Immune reaction of serum was much stronger than that of meat juice, which is compatible with ELISA results (Fig. 1 and 2). Although there was less titer of antibody in immunized meat juice compared with immunized serum, BIO-TAG is stably expressed in immunized meat juice and is distinguishable from non-immunized meat juice. Therefore, it is possible to identify meat juice immunized with BIO-TAG by Western blot analysis.

Based on the result of ELISA, dilution level of primary antibody for Western blot analysis was determined to be $1: 10,000$ since this concentration was high enough for detection of anti-BIO-TAG titers. However, with 1:100 dilution level, anti-BIO-TAG titers were detected even in non-immunized meat juice. This can be a false positive reaction when high concentration of antibody is applied in Western blot analysis. These results can be used as important information to screen an appropriate booster dose in rapid diagnosis kit.

The reason for setting the concentration of primary antibody as 1:10,000 shown in Fig. 3A and B is because 1:10,000 dilution in ELISA was high enough to discriminate the immunized meat juice from non-immunized one as shown in Fig. 4. Important thing is that non-immunized meat juice showing numerically the highest antibody-antigen reaction compared with immunized serum or meat juice at 1:100 dilution maybe caused by a false positive reaction with protein particles present in meat juice at lower dilution rate. These results should be reflected in future research for developing the diagnostic kit.

\section{Screening Hanwoo brand meat with rapid diagno- sis kit}

ELISA was tested on immunized- and non-immunized serum with primary antibody diluted by 1:1,000 to confirm that anti-BIO-TAG titers can be detected in Hanwoo administered with BIO-TAG vaccine. Although detection levels were lower than sheep titers showing in Fig. 1 and 2, anti-BIO-TAG titers in immunized Hanwoo (Hanwoo $\mathrm{A}, \mathrm{B}$ and $\mathrm{C})$ sera were greater $(p<0.05)$ than those in nonimmunized controls (Fig. 5). Although the number of subjects recruited in vaccination was relatively small, these results indicate that one dose of BIO-TAG immunization
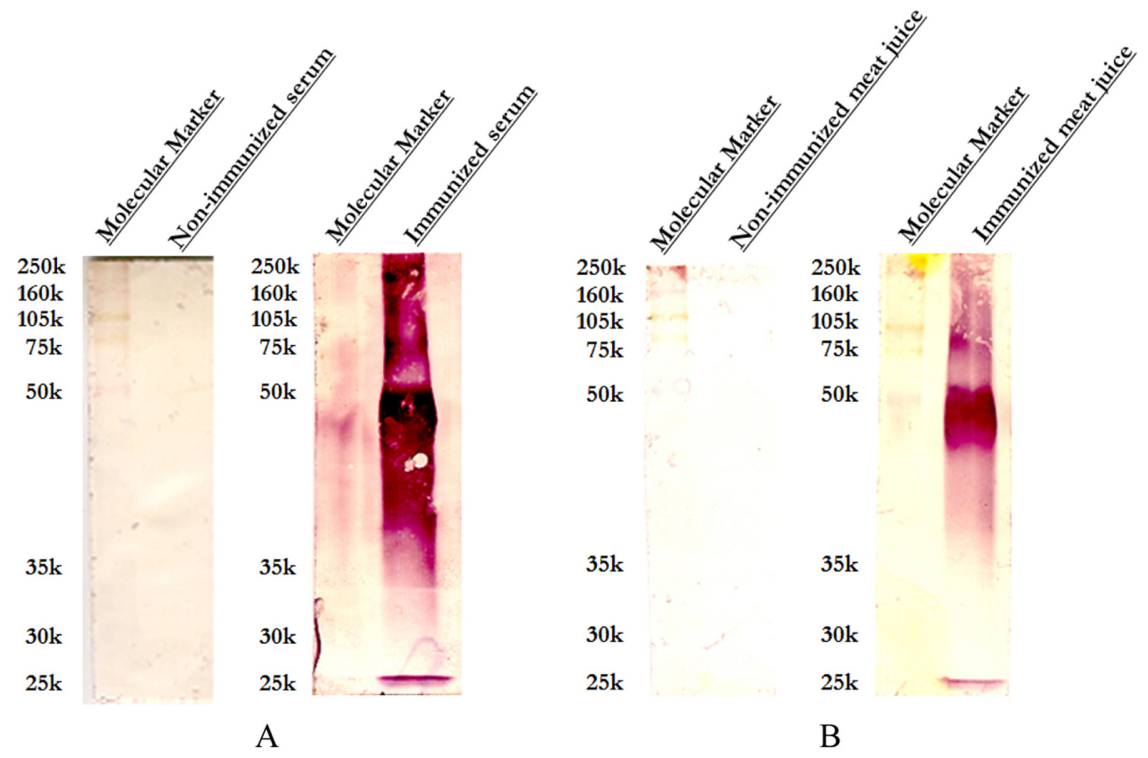

Fig. 3. Western immunoblotting using BIO-TAG (synthetic peptides) as an antigen and immunized serum (A) and immunized meat juice (B) as antibodies taken from sheep. Equivalent amount (10 ug) of ovalubumin conjugated BIO-TAG were loaded at each lane. Alkaline phosphatase conjugated anti-sheep IgG was used as a secondary antibody. 


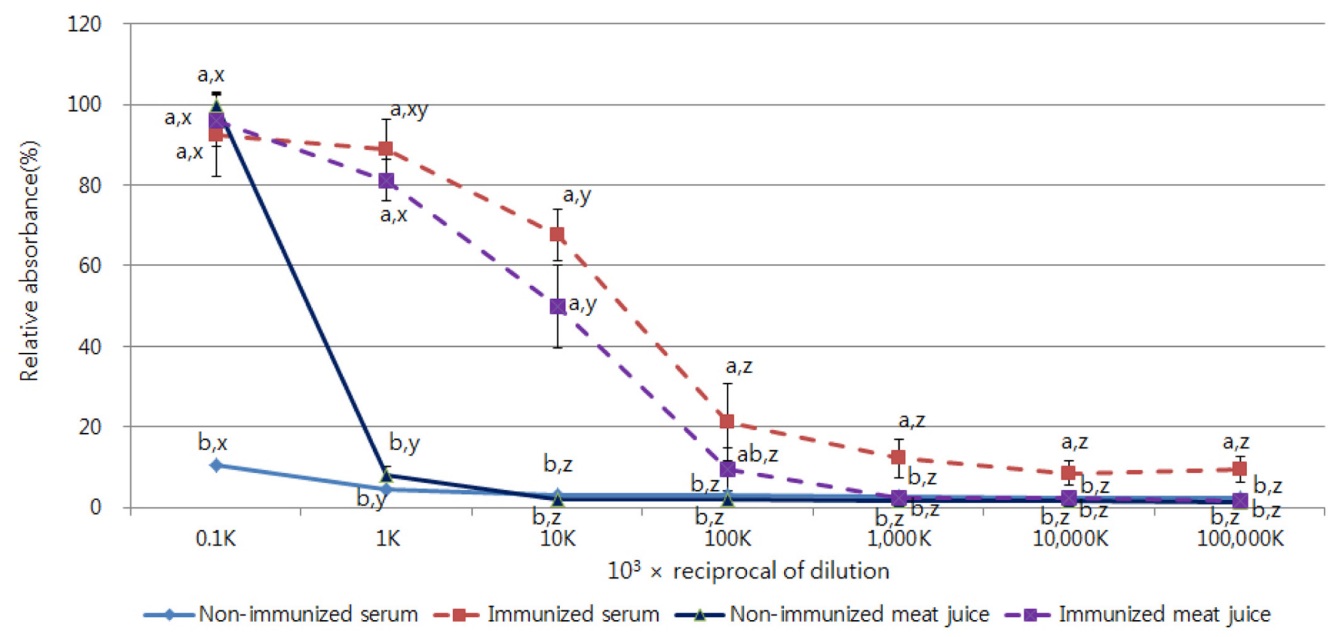

Fig. 4. Reactivity of non-immunized serum, antiserum and meat juices (non-immunized and immunized) in sheep against BIOTAG (synthetic peptides) depending on dilution ratio of primary antibody. The relative reactivity was expressed as percentage of the maximum optical density of non-immunized meat juice. Error bars are expressed as means \pm S.E. for 3 time repeats. Values with different letters a or $\mathrm{b}$ are significantly different among 4 treatments within the same dilution rate and $\mathrm{x}, \mathrm{y}$ or $\mathrm{z}$ are different between each dilution rates $(0.1 \mathrm{k}, 1 \mathrm{k}, 10 \mathrm{k}, 100 \mathrm{k}, 1,000 \mathrm{k}, 10,000 \mathrm{k}$ and 100,000 k) by the same treatment $(p<0.05)$.

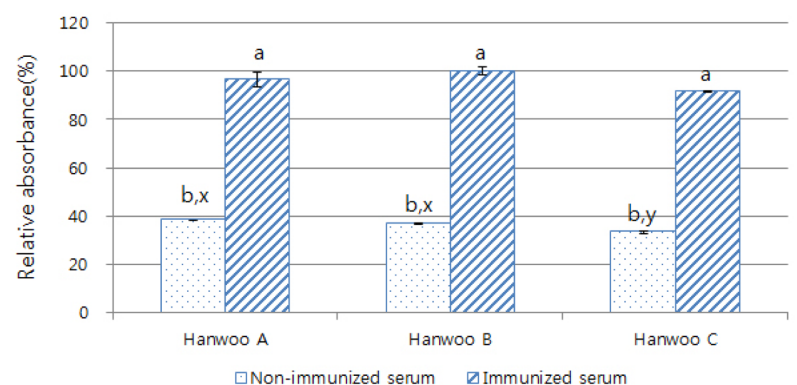

Fig. 5. Titers of immunized serum raised against BIO-TAG (synthetic peptides) in three Hanwoo (Korean Native Cattle) steers individuals immunized with antigen once. Error bars are expressed as means \pm S.E. for 3 time repeats. Values with different letters a or $b$ are significantly different between 2 treatments (non-immunized serum and immunized serum) and $\mathrm{x}$ or $\mathrm{y}$ are different between Hanwoo steers (Hanwoo A, Hanwoo B and Hanwoo C) by the same treatment $(p<0.05)$.

is enough for Hanwoo to possess the anti-BIO-TAG titers.

To check if vaccine was evenly distributed in animal body, antigen-antibody reaction for BIO-TAG in meat juice from different muscles including fore shank, loin, hind shank was analyzed (Fig. 6). Meat juice of loin and fore shank from immunized Hanwoo showed greater $(p<$ 0.05 ) antibody titer by $71.35 \%$ and $44.79 \%$, respectively, compared with those from non-immunized meat juice. However, antibody titer in hind shank from immunized meat juice was numerically but not statistically higher than non-immunized control. This result suggest that application of one vaccination may not be possible to fully

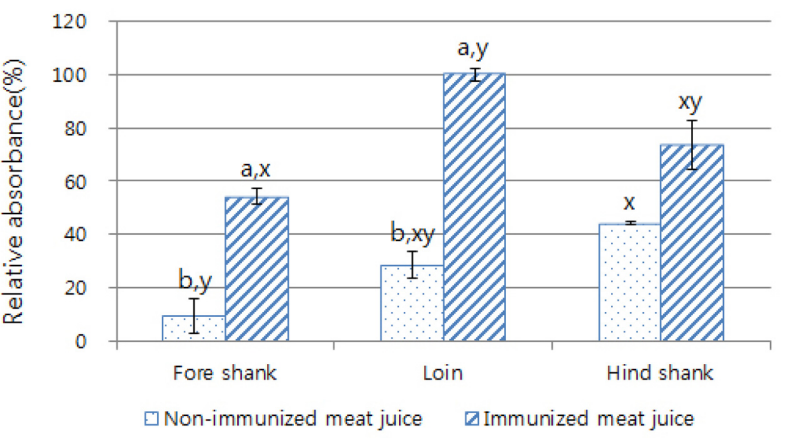

Fig. 6. Titers of meat juice raised against BIO-TAG (synthetic peptides) in three Hanwoo (Korean Native Cattle) steers individuals immunized with antigen once. Error bars are expressed as means \pm S.E. for 3 time repeats. Values with different letters $a$ or $b$ are significantly different between 2 treatments (non-immunized meat juice and immunized meat juice) and $\mathrm{x}$ or $\mathrm{y}$ are different between 3 meat cuts (fore shank, loin and hind shank) by the same treatment $(p<0.05)$.

discriminate each part of meat of Hanwoo. Therefore, it will be necessary to develop more effective immunization methods by investigating antibody production ratio of various parts of meat, adjusting the level of antigen and adjuvant and boosting immunization.

To optimize the dilution factor, we performed dot-ELISA with various combination levels of serum, meat juice and BIO-TAG (Fig. 7). Immunized serum placed onto line A, as a positive control, showed strong signals irrespective of the antigen levels including $2,0.2$ and $0.02 \mathrm{mg} / \mathrm{mL}$ BIOTAG. However, non-immunized meat juice placed on line 


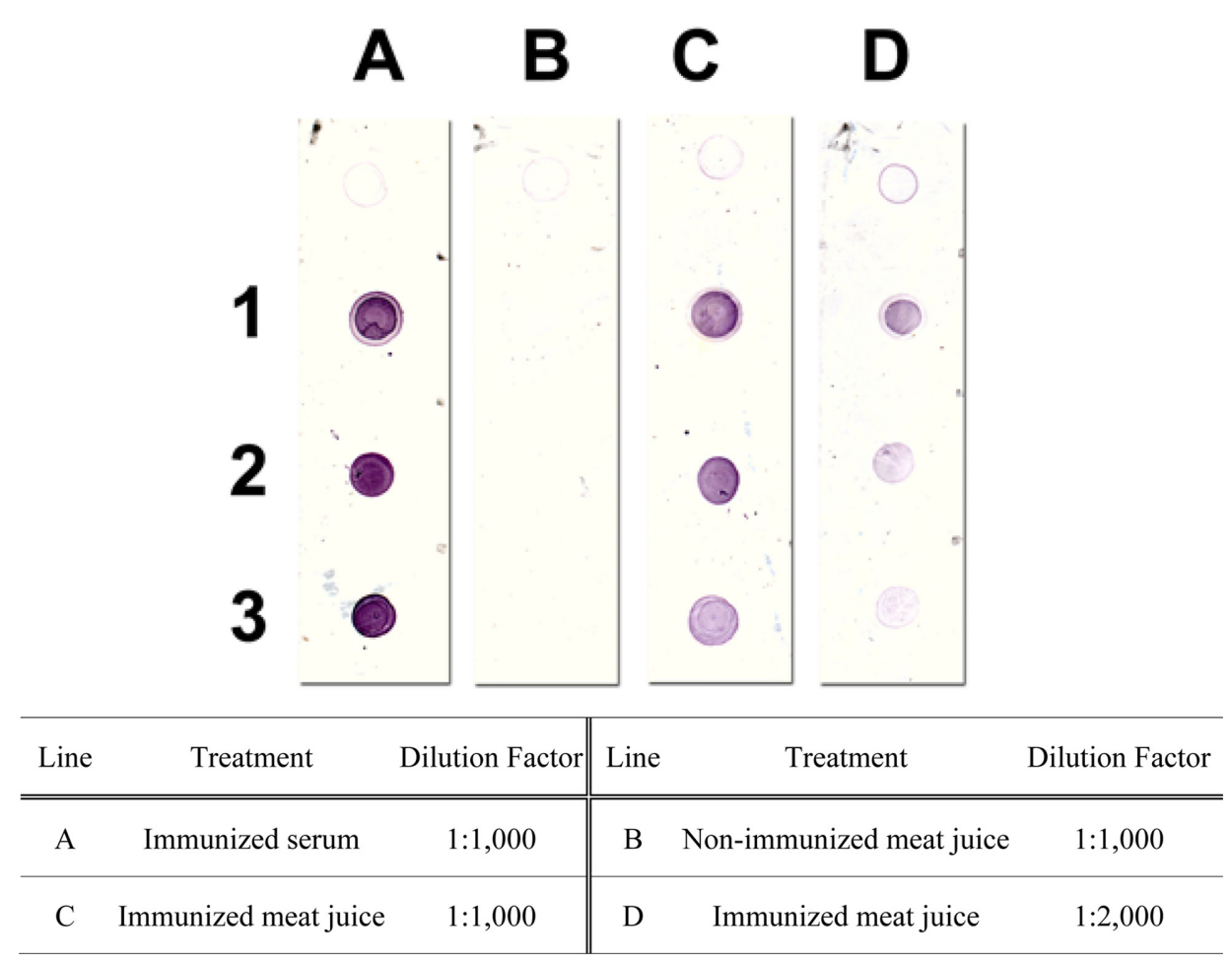

Fig. 7. The result of dot-ELISA. Various concentration of BIO-TAG (conjugated with ovalbumin) was dotted onto the nitrocellulose membrane: row $1(2 \mathrm{mg} / \mathrm{mL})$, row $2(0.2 \mathrm{mg} / \mathrm{mL})$, row $3(0.02 \mathrm{mg} / \mathrm{mL})$. Different levels of immunized or non-immunized serum and meat juice was then applied onto the BIO-TAG, which was shown in the table. Anti-bovine IgG-horseradish peroxidase conjugated secondary antidoby was used for color detection.

$\mathrm{B}$ had no response at any concentration level. Immunized meat juice on line $\mathrm{C}$ showed stronger response to BIOTAG antigen compared with $2 \times$ diluted meat juice on line D. The intensity of purple color was decreased as BIOTAG antigen was decreased from 2 to $0.02 \mathrm{mg} / \mathrm{mL}$ in both line $\mathrm{C}$ and $\mathrm{D}$. Although the intensity of immune response was lower in line D compared to line $\mathrm{C}$, antigen concentration used in raw 1 ( $2 \mathrm{mg} / \mathrm{mL}$ BIO-TAG) was sufficient to distinguish the immunized meat from non-immunized control.

In conclusion, these results support our hypothesis that we can identify Hanwoo brand meat by vaccination with BIO-TAG and distinguish the immunized Hanwoo by detecting the immune reaction. This technology can be applied into immunostrip for developing the rapid diagnosis kit. Hereafter, if rapid diagnosis kit for rooting out counterfeit sales is developed, it is eventually expected to be applied as the major protective device for the high quality Hanwoo brand meat in the domestic market. In addition, BIO-TAG is a naturally occurring protein and safe to be used as a food so that it meets the needs of nowadays consumers interest in health and safety. To this end, further research will be necessary to screen the optimal reaction condition and develop prototypes as well as obtain the candidates of BIO-TAG followed by performing the large-scale validation tests.

\section{Acknowledgements}

This work was supported by the 2009 Yeungnam University Research Grant.

\section{References}

1. Baek, K. H., Choi, C. W., Choi, C. B., and Lee, B. S. (2007) Passive immunization approach to reduce body fat in pigs using fat-specific polyclonal antiserum. Asian-Aust. J. Anim. Sci. 20, 1594-1599.

2. Baek, K. H., Kwak, T. H., Oh, Y. S., Choi, C. W., Jung, K. K., and Choi, C. W. (2005) Studies on the development and utilization of polyclonal antibodies against swine adiocyte plasma membrane proteins. J. Anim, Sci. Technol (Kor.). 47, 19-28.

3. Benkirane, N., Guichard, G., Van Regenmortel, M. H. V., Briand, J., and Muller, S. (1995) Cross-reactivity of antibodies to retro-inverso peptidomimetics with the parent protein histone H3 and chromatin core particle. J. Biol. Chem. 270, 1192111926.

4. Briand, J. P., Benkirane, N., Guichard, G., Newman, J. F. E., Van Regenmortel, M. H. V., Brown, F., and Muller, S. (1997) 
A retro-inverso peptide corresponding to the GH loop of footand-mouth disease virus elicits high levels of long-lasting protective neutralizing antibodies. Proc. Natl. Acad. Sci. 94, 12545-12550.

5. Cho, H. W. and Ahn, B. H. (2006) Effects of dietary vitamin E levels on carcass characteristics, vitamin E concentration of Longissimus muscle and lipid oxidation in Hanwoo steers. J. Anim. Sci. Technol. (Kor.). 48, 827-838.

6. Cho, W. M., Cho, Y. M., Hong, S. K., Jeong, E. S., Lee, J. M., and Yoon, S. K. (2000) Effects of feeding whole crop barley silage on growth performance, feed efficiency and meat quality in Hanwoo steers. J. Anim. Sci. Technol. (Kor.). 42, 181-188.

7. Choi, C. W., Baek, K. H., Choi, C. B., Oh, Y. K., and Hong, S. G. (2010) Development of polyclonal antibodies to abdominal and subcutaneous adipocytes for producing fat-reduced high quality pork. Korean J. Food Sci. An. 30, 87-94.

8. Choi, C. W., Kim, Y. H., Kim, S. J., Song, M. G., Kwon, E. G., Oh, Y. K., Hong, S. G., Choi, S. H., and Baek, K. H. (2008) Development of polyclonal antibodies to abdominal and subcutaneous adipocytes for fat-reduced Hanwoo beef production. Korean J. Food Sci. An. 28, 651-659.

9. Chung, E. R. and Chung, K. Y. (2004) Identification of beef breed using DNA marker of coat color genes. Korean J. Food Sci. An. 24, 355-360.

10. IEDB (Immune Epitope Database) and Analysis Resource (2012) User Documentation. La Jolla Institute for Allergy and Immunology. 9420 Athena Circle, La Jolla, CA 92037, USA. Available from: http://tools.immuneepitope.org/main/index. html

11. Kim, D. G., Jung, K. K., Sung, S. K., Choi, C. B., Kim, S. G., Kim, D. Y., and Choi, B. J. (1996) Effects of castration on the carcass characteristics of Hanwoo and Holstein. J. Anim. Sci. Technol. (Kor.). 38, 239-248.

12. Kim, D. G., Jung, K. K., Sung, S. K., Choi, C. B., Kim, S. K., Kim, D. Y., and Choi, B. K. (1996) Effects of age in the carcass characteristics of Hanwoo and Holstein steers. J. Anim. Sci. Technol. (Kor.) 38, 268-274.

13. Kim, K. H., Lee, J. H., Oh, Y. G., Kang, S. W., Lee, S. C., Park, W. Y., and Ko, Y. D. (2005) The optimal TDN levels of concentrates and slaughter age in Hanwoo steers. J. Anim. Sci. Technol. (Kor.). 47, 731-744.

14. Kramer, A., Stigler, R., Knaute, T., Hoffmann, B., and Schneider-Mergener, J. (1998) Stepwise transformation of a cholera toxin and a p24(HIV-1) epitope into D-peptide analogs. Protein. Eng. 11, 941-948.

15. Lee, K. C., Park, N. H., Jeong, J., Lee, S. S., Oh, Y. S., Baek, K. H., Jung, K. K., and Choi, C. B. (2004) Effects of castration and slaughtering ages on Physico-chemical characteristics of Hanwoo M. Longissimus dorsi. J. Anim. Sci. Technol.
(Kor.). 46, 165-172.

16. Lee, K. J. and Watson, R. D. (2002) Antipeptide antibodies for detecting crab (Callinectes sapidus) molt-inhibiting hormone. Peptides 23, 853-862.

17. Lee, M. Y. (1998) Effects of body weight and age on carcass traits of Hanwoo (Korean cattle) steer. J. Anim. Sci. Technol. (Kor.). 40, 221-226.

18. Lee, S. S., Jung, J. K., Park, N. H., and Won, Y. S. (1997) Effects of castration on the carcass characteristics and serum metabolites in Korean cattle. J. Anim. Sci. Technol. (Kor.). 39, 145-154.

19. Macedo-Silva, A., Barbosa, S. F. C., Alkmin, M. G. A., Vaz, A. J., Shimokomaki, M., and Tenuta, A. A. (2000) Hamburger meat identification by dot-ELISA. Meat Sci. 56, 189-192.

20. Mohri, H., Asakura, Y., Fukushima, J., Kawamoto, S., Okubo, T., and Okuda, K. (1997) Synthetic peptide from the V3 loop consensus motif with a potent anti-HIV activity inhibits ristocetin-mediated vWF-GPIb interaction. Peptides 18, 12891293.

21. Nargi, F., Kramer, E., Mezencio, J., Whetstone, C., Van Regenmortel, M. H. V., Briand, J. P., Muller, S., and Brown, F. (1999) Protection of swine from foot-and-mouth disease with one dose of an all-D retro peptide. Vaccine 17, 2888-2893.

22. Paek, B. H., Kang, S. W., Cho, Y. M., Cho, W. M., Yang, C. J., and Yun, S. G. (2005) Effects of substituting concentrates with dried leftover food on growth and carcass characteristics of Hanwoo steers. Asian-Aust. J. Anim. Sci. 18, 209-213.

23. Song, M. K. and Choi, Y. I. (1994) Effect of feeding method, supplementation of Yellow Grease and duration of feeding on carcass characteristics and meat quality of Korean native bulls. Korean J. Anim. Nutr. Feed. 18, 30-37.

24. Song, M. K., Kim, N. S., Chung, C. S., Choi, Y. I., Won, Y. S., Chung, J. K., and Choi, S. H. (1998) Effect of feeding level of concentrates on growth performance and fatty acid composition of adipose tissues at various locations in Hanwoo bulls. J. Anim. Sci. Technol. (Kor.). 40, 485-498.

25. Sung, S. K., Jung, K. K., Choi, C. B., Kim, D. G., Kim, S. G., Kim, D. Y., and Choi, B. J. (1996) Effects of castration and age on the carcass composition and retail yields of Hanwoo and Holstein. J. Anim. Sci. Technol. (Kor.). 38, 261-267.

26. Tooyama, I., Abe, H., Renda, T. G., and Kimura, H. (2000) Production and immunohistochemical application of antiserum against Tyr-D-Ala-Phe, a N-terminal tripeptide common to dermorphin/deltorphin family. Peptides 21, 1649-1655.

27. Van Rgenmortel, M. H. V. and Muller, S. (1998) D-peptides as immunogens and diagnostic reagents. Curr. Opin. Biotechnol. 9, 377-382.

(Received 2014.1.14/Revised 2014.4.14/Accepted 2014.5.12) 